\title{
Soft X-rays of AGN: Emission from Accretion Disks?
}

\author{
R. Staubert, T. Dörrer, C. Müller \\ Institut für Astronomie und Astrophysik, Astronomie, Universität \\ Tübingen, Germany \\ P. Friedrich, H. Brunner \\ Astrophysikalisches Institut Potsdam, Germany
}

\section{Introduction}

Soft X-ray spectra of many Active Galactic Nuclei (AGN) show structure which suggests excess emission at low energies, mostly below $1 \mathrm{keV}$. This was confirmed by the ROSAT spectra (0.1-2.4 keV) AGN in our samples which generally have steeper power law spectra than the canonical index of 0.7 . The soft excess component may be the high energy tail of the big blue bump which in turn may be due to the integrated emission from an accretion disk around the central black hole.

We discuss results of our spectral analysis of two different samples of AGN: 1) QSO/Seyfert-I from the ROSAT All Sky Survey (RASS) and 2) radio-quiet QSO from ROSAT Pointed Observations. The ROSAT data are combined with UV Data from IUE and hard X-ray data from various hard X-ray missions.

The soft X-ray component - in combination with UV and hard X-ray data is described in terms of thermal emission from a thin $\alpha$-accretion disk, including Comptonization and relativistic corrections. We have developed disk models of increasing complexity. Constraints on the physical parameters of the systems black hole mass, accretion rate, viscosity and inclination angle - are derived by fitting these disk models (including the galactic $\mathrm{N}_{\mathrm{H}}$ and two power law components describing the IR to optical/UV continuum and the hard X-ray spectrum, respectively) to the observational data.

\section{QSO and Seyfert-I galaxies from the ROSAT All Sky Survey}

Sample 1: 1) QSO and Seyfert-I from the Véron-Cetty \& Véron catalogue, 2) IUE low dispersion spectra available, 3) RASS detection in each of the energy bands: $0.07-0.4 \mathrm{keV}, 0.4-1.0 \mathrm{keV}, 1.0-2.4 \mathrm{keV}$. From the 420 IUE-selected AGN finally 89 remained. Hard X-ray data were taken from the literature.

Accretion disk model 1 is based on the $\alpha$-accretion disk model by Shakura and Sunyaev (1973) and Novikov and Thorne (1973), as coded by Dörrer (1991): 1) Standard geometrically thin $\alpha$-accretion disk, 2) Vertically averaged structure including relativistic corrections, 3) Eddington approximation for radiative transfer with saturated Comptonization included (Czerny \& Elvis 1987), 4) General relativistic effects incorporated by 'calculation of the Cunningham transfer function (Cunningham 1975, Speith et al. 1995). See also Dörrer et al. (1992). 


\section{Radio-quiet QSO from the ROSAT pointed observation phase}

Sample 2: 1) QSO from the Véron-Cetty \& Véron and the Hewitt \& Burbidge catalogues. 2) Radio to optical spectral index $\alpha_{\mathrm{RO}}$ flatter than 0.3. 3) Public IUE and ROSAT PSPC data. 4) At least 200 PSPC source counts. The final sample defined in this way consists of 31 objects.

Accretion disk model 2 (Dörrer et al. 1996) has the following additional features: 1) Self-consistent description of the vertical structure and radiation field of the disk around a Kerr black hole by solution of hydrostatic equilibrium, radiative transfer, energy balance, and equation of state. 2) Viscosity entirely due to turbulence. 3) Multiple Compton scattering treated in Fokker-Planck approximation using the Kompaneets operator.

The parameters of both models are: mass $M$ of the central black hole, accretion rate $\dot{M}$ (in units of $\dot{M}_{\mathrm{Edd}}$ ), viscosity parameter $\alpha$ and inclination angle $\Theta$ of observer with respect to the disk axis. Our calculations are for a bare disk without the ad-hoc addition of a hot corona.

\section{Results and Conclusion}

The fitted accretion rates using our model 2 (less than 0.3 ) are generally lower than those from model 1 and are consistent with the thin disk approximation. This is mainly because model 2 also takes into account the temperature gradient in the vertical direction of the disk. This means, that the local spectra differ from the blackbody even in the optically thick case, leading to harder spectra. The viscosity parameters required on the other hand are relatively high.

Our general conclusion is therefore that emission from an accretion disk can in many cases account for the observed spectral features, such as the soft $\mathrm{X}$-ray excess. Detailed publications about the analysis of both samples are in preparation (Friedrich et al. 1996, Brunner et al. 1996).

\section{References}

Brunner, H., Müller, C., Friedrich, P., Dörrer, T., Staubert, R. 1996, in prep. Cunningham, C. T. 1975, ApJ, 202, 788

Czerny, B., and Elvis, M. 1987, ApJ, 321, 305

Dörrer, T. 1991, Diploma thesis University of Tübingen

Dörrer, T., Friedrich, P., Brunner, H., Staubert, R. 1992, MPE Rep 235, 235

Dörrer, T., Riffert, H., Staubert, R., Ruder, H. 1996, A\&A, 311, 69

Friedrich, P., Brunner, H., Dörrer, T., Staubert, R. 1994, IAU Symp. 159, 374

Friedrich, P., Dörrer, T., Brunner, H., Staubert, R. 1996, in prep.

McDowell, J. C. 1992, ApJ, 384, 62

Novikov, I.D., and Thorne, K.S. 1973, in Black Holes, ed. C. DeWitt and B. DeWitt (New York: Gordon \& Breach), 343

Shakura, N. I., and Sunyaev, R. A. 1973, A\&A, 24, 337

Speith, R., Riffert, H., Ruder, H. 1995, Comp. Phys. Comm., 88, 109 